\title{
Espectroscopia do visível e infra-vermelho próximo para estimar sólidos solúveis e firmeza de polpa em função da época de colheita em pêssegos
}

\section{Spectroscopy of the visible and near infrared to evaluate soluble solids and flesh firmness in peach according to harvest season}

\author{
Débora Leitzke Betemps ${ }^{1 *}$; José Carlos Fachinello²; Simone Padilha Galarça ${ }^{3}$; \\ Nicácia Portela Machado ${ }^{4}$; Damiano Remorini ${ }^{5}$; Rossano Massai ${ }^{6}$; Giovanni Agati ${ }^{7}$
}

\section{Resumo}

O objetivo deste estudo foi estabelecer modelos de calibração para estimar sólidos solúveis e firmeza da polpa de forma não destrutiva em pêssegos 'Chimarrita', 'Maciel', 'Eldorado' e 'Jubileu' observando o efeito da época da colheita das frutas sobre estes modelos. Para isto, no primeiro ano de execução foi utilizado o espectrofotômetro NIR-Case (SACMI) no qual foram estabelecidas as equações de calibração para cada cultivar a partir das avaliações por métodos não destrutivos e destrutivos e, posteriormente, testou-se o desempenho destes modelos através do procedimento da validação com a observação dos resultados coeficiente de determinação $\left(\mathrm{R}^{2}\right)$ e erro padrão dos valores previstos (SEP). No segundo ano de experimento utilizando o mesmo equipamento e metodologia, datas de colheitas ao longo da maturação dos pêssegos foram estipuladas e nestas foram desenvolvidos modelos de calibração para cada cultivar estudada. De acordo com os resultados obtidos, conclui-se que os modelos propostos para avaliação da qualidade das frutas foram satisfatórios para predizer o teor de sólidos solúveis nas cultivares em estudo, e o mesmo resultado não foi observado em relação à firmeza de polpa no primeiro ano de avaliação. No segundo ano observou-se que os valores dos parâmetros estatísticos referentes a validação dos modelos estabelecidos se alteram ao longo das diferentes épocas de colheita, mas com valores de $\mathrm{R}^{2}$ e SEP aceitáveis para estimar os teores de sólidos solúveis em todas as cultivares e para o parâmetro firmeza de polpa apenas para as cultivares Eldorado e Jubileu os valores foram considerados satisfatórios.

Palavras-chave: Prunus persica, avaliação da qualidade, métodos não destrutivos

\footnotetext{
Abstract

This study aimed to establish calibration models to assess soluble solids contents and flesh firmness in a non destructive way in peaches from 'Chimarrita', 'Maciel', 'Eldorado' and 'Jubileu' varieties,

${ }^{1}$ Prof $^{a}$ Adj. da Universidade Federal da Fronteira Sul, UFFS, Cerro Largo, RS. E-mail: debora.betemps@uffs.edu.br

2 Prof. Titular da Universidade Federal de Pelotas, UFPel, Pelotas, RS. E-mail: jfachi@ufpel.tche.br

${ }^{3}$ Extensionista, Eng ${ }^{\mathrm{a}}$ Agr ${ }^{\mathrm{a}}$, Emater/Ascar, Piratini, RS. E-mail: sgalarca@superig.com.br

${ }^{4}$ Bolsista CNPQ, UFPel, Pelotas, RS. E-mail: nicacia@gmail.com

${ }_{5}$ Pesquisador, Universidade de Pisa, "Dipartimento di Scienze Agrarie, Alimentari e Agro-ambientali”, UNIPI, Pisa, Itália. E-mail: damiano.remorini@unipi.it

6 Prof. da Universidade de Pisa, "Dipartimento di Scienze Agrarie, Alimentari e Agro-ambientali”, UNIPI, Pisa, Itália. E-mail: rossano.massai@unipi.it

7 Pesquisador, Istituto di Fisica Applicata “Nello Carrara”_"Centro Nazionale di Ricerca”, CNR, Firenze, Itália. E mail: g.agati@ ifac.cnr.it

* Autor para corespondência
} 
watching the harvest season effect on these models. Thereunto, in the first year of execution it was used the spectrophotometer Nir-Case (SACMI) in order to determine the calibrations equations for each cultivar from the non destructive and destructive evaluations. After that the performance of these models were evaluated through the procedure of validation with the remark of the results of determination coefficient $\left(\mathrm{R}^{2}\right)$ and standard error of the predicted values (SEP). In the second year of experiment, using the same equipment and methodology, harvest dates along the peach maturation period were stipulated and calibrations models were developed for each cultivar studied. According to the results it can be concluded that the proposed models for evaluation of quality of fruit were satisfactory and the same result was not observed in the flesh firmness in the first year. In the second year it was observed that the statistics parameters values relative to the validation of the established models change along different periods during harvest seasons. However, the $\mathrm{R}^{2}$ and SEP values were considered acceptable to estimate the content of soluble solids in all cultivars. The parameter of flesh firmness was only satisfactory for the cultivars Eldorado and Jubileu.

Key words: Prunus persica, quality evaluation, non-destructive methods

\section{Introdução}

Em pêssegos (Prunus persica L. Batsch), a colheita é normalmente realizada com base na cor de fundo da epiderme e no tamanho da fruta. A firmeza da polpa (FP) e o conteúdo de sólidos solúveis (SS) também são utilizados para avaliar a maturação e a qualidade, entretanto estas análises requerem a utilização de um número limitado de amostras além da destruição das frutas (NOFERINI et al., 2008).

Técnicas não destrutivas como a espectroscopia no infravermelho próximo (Near infrared-NIR) tem sido proposta para avaliar características representativas de qualidade em frutas pois permite rápida execução das medições, não necessita de qualquer preparação das amostras, tem potencial para estimar vários atributos de qualidade simultaneamente além da possibilidade de repetir as medições nas mesmas amostras (HUANG et al., 2008).

No entanto, esta técnica depende de modelos de calibração para prever os parâmetros e estes desempenham um papel importante em trabalhos com espectroscopia do infravermelho, pois, é através do modelo utilizado que se determina o comportamento do sistema como um todo (LIN; YING, 2009). Uma vez determinado o modelo que será utilizado como calibração é necessário que se valide sua capacidade de predição. Este procedimento é especialmente relevante para a verificação do nível de ajuste do modelo no momento da previsão de novas amostras
(BEEBE; PELL; SEASHOLTZ, 1998). Um modelo é construído e validado pela utilização de vários conjuntos de amostras. O primeiro é o conjunto de calibração usado para estabelecer o modelo. O segundo conjunto corresponde a validação, usado para avaliar a capacidade do modelo para estimar amostras desconhecidas. O conjunto de calibração e de validação são independentes e devem consistir amostras de lotes distintos (ROGGO et al., 2007).

A escolha dos componentes que irão compor as amostras é de extrema importância, pois é necessário incluir no modelo espectros correspondente a frutas extremamente heterogêneas nos valores dos diversos parâmetros de maturação avaliados (COSTA et al., 1999). Entretanto, na prática, estipular as frutas que irão compor a amostra para a calibração não é uma tarefa fácil, pois a maturação, principalmente nas climatéricas como é o caso de pêssegos, normalmente é concentrada em poucas semanas tornando difícil contemplar todas as classes de maturação em um mesmo período.

Trabalhos de calibrações envolvendo diferentes épocas de colheita já foram testados em culturas como melões e abacaxis (GUTHRIE; WEDDING; WALSH, 1998), maçãs (MCGLONE; KAWANO, 1998) e quivis (BOBELYN et al., 2010) e onde os autores constataram que calibrações realizadas em diferentes épocas de colheita apresentaram baixos coeficientes de determinação quando a variabilidade das frutas não são incorporadas aos modelos de 
calibração NIR para os parâmetros firmeza, teor de matéria seca e sólidos solúveis.

No Brasil, existem poucos estudos de pesquisa sobre a aplicação da espectroscopia do infravermelho em análises de qualidade de frutas e como se trata de um método rápido e que não gera nenhum tipo de resíduo, torna-se importante a execução de trabalhos que possibilitem estabelecer as potencialidades e os pontos críticos da utilização desta técnica na avaliação da qualidade. Deste modo, o objetivo deste estudo foi estabelecer modelos de calibração para estimar os teores de sólidos solúveis e firmeza da polpa de forma não destrutiva em pêssegos 'Chimarrita', 'Maciel', 'Eldorado' e 'Jubileu' observando o efeito da época da colheita das frutas sobre estes modelos.

\section{Material e Métodos}

As frutas das cultivares Chimarrita, Maciel, Eldorado e Jubileu provenientes do pomar didático do Centro Agropecuário da Palma/FAEM- UFPel localizado no município de Capão do Leão/RS (Latitude $31^{\circ} 52^{\prime}$ 00” S; Longitude 52 $21^{\circ}$ ' 24" W Greenwich;Altitude: 13,24 m.) foram utilizadas para a execução deste experimento no qual constaram de avaliações em duas safras agrícolas (2009 e 2010).

Para a execução do trabalho realizaram-se calibrações e validações específicas para cada cultivar. Os primeiros modelos de calibração foram realizados no ano de 2009 e utilizou-se um espectrofotômetro próprio para o uso em frutas (NIR-Case), sendo as frutas obtidas de diferentes plantas e com diferentes estádios de maturação. Para todas as cultivares relatadas neste primeiro ano de estudo foram estipuladas 120 frutas para compor a amostra de calibração e 50 frutas para a amostra de validação.

As avaliações não destrutivas (NIR-Case) e destrutivas (referência) foram realizadas na porção equatorial (lados opostos A e B) das frutas, sendo elas: firmeza da polpa (FP) e teor de sólidos solúveis (SS).
As avaliações não destrutivas foram realizadas com o instrumento espectrofotômetro NIR CASE (SACMI Imola, Itália). Este equipamento efetua as medições através dos princípios da interactância da luz. A fonte de luz é constituída por oito lâmpadas de halógeno e o comprimento de onda varia entre 600 a 1000 nanômetros $(\mathrm{nm})$. O tempo de medição varia entre 6 mili-segundos e 2 segundos. Antes de efetuar as medições, foram feitos os procedimentos de configuração e calibração do aparelho (luz branca e negra).

Após a geração dos espectros neste equipamento, considerando os mesmos lados (A e B), as frutas passaram para a fase das análises destrutivas.

As avaliações destrutivas (referência) foram realizadas separadamente considerando os mesmos lados das frutas (lados A e B). A determinação da firmeza da polpa (FP) foi realizada com um penetrômetro digital com ponteira de $8 \mathrm{~mm}$ de diâmetro (modelo 53205, TR, Forli, Italy), sendo os resultados expressos em $\mathrm{Kgf} / \mathrm{cm}^{2}$ e o conteúdo de sólidos solúveis (SS) foi mensurado com refratômetro digital (Atago PR32) e os resultados expressos em ${ }^{\circ}$ Brix.

Os espectros gerados pelo NIR-Case e os valores obtidos pelo método de referência das amostras foram utilizados para a obtenção das calibrações, as quais foram calculadas utilizando o software NCS (NIR CALIBRATION SOFTWARE) Vers. 3.0 R C 1. As análises matemáticas (análise multivariada) bem como o algoritmo usado para o desenvolvimento dos modelos de calibração são partes do software que acompanha o equipamento. As equações de calibração foram confrontadas através dos parâmetros estatísticos: coeficiente de regressão múltipla $\left(\mathrm{R}^{2}\right)$ e erro 'standard' de calibração (SEC).

Um novo conjunto de amostras (predição), com as frutas devidamente identificadas foi submetido ao NIR-Case, (utilizando a calibração realizada na descrição anterior), onde se obteve os valores preditos pelo equipamento para estas frutas, 
que foram confrontados com os valores reais. A precisão das equações de calibração foi confrontada através dos parâmetros estatísticos coeficiente de determinação $\left(\mathrm{R}^{2}\right)$, e o erro padrão dos valores previstos (SEP- Standard error of prediction), com o auxílio do programa estatístico Sigmapolt 10.0.

No segundo ano, safra 2010/11, para tornar os modelos mais robustos, foram incluídos os modelos de calibração do ano anterior (2009), específicos para cada cultivar e a estes foram acrescentados novos espectros do ano de 2010/11. Para a avaliação da influência da data de colheita no processo de calibração, para cada cultivar foram estipuladas diferentes datas de colheita ao longo da maturação, com intervalos de aproximadamente sete dias (tabela 1) e, em cada data foram construídos modelos de calibração específicos para cada cultivar. As frutas foram provenientes do pomar acima citado e foram coletadas em diferentes estádios de maturação sendo que para todas as cultivares, em cada data de colheita, o número de amostras foi de 230 frutas para calibração e 100 amostras para a predição. Em cada data de colheita, para os modelos de calibração constaram de 350 espectros devido a inclusão dos espectros do ano anterior.

Tabela 1. Cultivares e datas de colheita estipuladas para a avaliação do efeito da época de colheita sobre os modelos de calibração. UFPel, Pelotas,RS, 2013.

\begin{tabular}{ccccc}
\hline Cultivar & Data 1 & Data 2 & Data 3 & Data 4 \\
\hline Chimarrita & $07 / 12 / 2010$ & $14 / 12 / 2010$ & $21 / 12 / 2010$ & - \\
Maciel & $18 / 12 / 2010$ & $24 / 12 / 2010$ & $31 / 12 / 2010$ & - \\
Eldorado & $19 / 12 / 2010$ & $24 / 12 / 2010$ & $28 / 12 / 2010$ & $05 / 01 / 2011$ \\
Jubileu & $19 / 12 / 2010$ & $24 / 12 / 2010$ & $28 / 12 / 2010$ & $05 / 01 / 2011$ \\
\hline
\end{tabular}

Fonte: Elaboração dos autores.

Foi utilizada a mesma metodologia descrita anteriormente para o desenvolvimento do trabalho de calibração e predição do segundo ano (avaliações não destrutivas e destrutivas). Os valores preditos pelo equipamento e os valores de referência foram confrontados através dos parâmetros estatísticos anteriormente descritos.

\section{Resultados e Discussão}

Foram estabelecidas calibrações e validações específicas para cada cultivar pois tratando-se de cultivares de pêssegos de polpa branca (Chimarrita) e de polpa amarela (Maciel, Eldorado e Jubileu) bem como com diferentes épocas de maturação, era fundamental respeitar as especificidades de cada cultivar para diminuir os efeitos nos espectros e, de acordo com Peirs et al. (2002) e Zerbini (2006), para cada espécie de fruto e variedade é necessário um novo modelo de calibração. Estes modelos devem ser baseados em grandes conjuntos de dados, incluindo frutos de diferentes pomares, épocas de colheita, sistemas de produção, entre outros.

Os valores obtidos para a calibração das cultivares Chimarrita, Maciel, Eldorado e Jubileu originados a partir de regressão múltipla (Tabela 2) apresentam variações importantes entre as cultivares e os parâmetros de qualidade avaliados. Neste trabalho os modelos de calibração apresentaram coeficientes aceitáveis (acima de 0,85) e valores baixos para os erros (SEC) para os parâmetros avaliados nas diferentes cultivares trabalhadas. 
Tabela 2. (A) Coeficientes de regressão múltipla $\left(\mathrm{R}^{2}\right)$, erro padrão de calibração (SEC)(valores em Brix e $\left.\mathrm{kgf} / \mathrm{cm}^{2}\right) \mathrm{e}$ valores médios ( $\mathrm{em}^{\circ}$ Brix e $\mathrm{kgf} / \mathrm{cm}^{2}$ ) referentes as calibrações, obtidas pelo software Nir-Case Sacmi® (NCS), dos parâmetros sólidos solúveis e firmeza de polpa de pêssegos das cultivares Chimarrita, Maciel, Eldorado e Jubileu (n:120 frutas) produzidas na região de Pelotas/RS no ano de 2009. UFPel, Pelotas,RS, 2013.

\begin{tabular}{lccc}
\hline \multicolumn{1}{c}{ Cultivar } & \multicolumn{1}{c}{ (A) Calibração/ Sólidos Solúveis } \\
\hline Chimarrita & $0,85^{* *}$ & SEC & Valores Médios (SS) \\
Maciel & 0,94 & 0,48 & 9,90 \\
Eldorado & 0,95 & 0,35 & 10,85 \\
Jubileu & 0,91 & 0,42 & 12,65 \\
\hline \multicolumn{4}{c}{ (A) Calibração/ Firmeza de polpa Valores Médios (FP) } \\
\hline Chimarrita & $0,90^{* *}$ & 0,48 & 11,09 \\
Maciel & 0,95 & 0,38 & 3,29 \\
Eldorado & 0,85 & 0,79 & 3,18 \\
Jubileu & 0,86 & 0,40 & 3,12 \\
\hline
\end{tabular}

$* * \mathrm{p}=0,001$.

Fonte: Elaboração dos autores.

A capacidade destes modelos de calibração prever resultados em amostras futuras foi avaliada com a validação dos modelos de calibração e para o parâmetro sólido solúvel observou-se que as calibrações foram válidas e que os parâmetros estatísticos coeficiente de determinação $\left(\mathrm{R}^{2}\right)$ e erro padrão dos valores previstos (SEP) (Tabela 3) apresentam comportamentos distintos de acordo com a cultivar. Para a predição das calibrações foram determinados os coeficientes de determinação de 0,82 para a cultivar Chimarrita, 0,84 para Maciel, 0,78 para Eldorado e 0,69 para a cultivar Jubileu. Segundo Scaritporn et al. (2008), coeficiente inferiores a 0,70 não são considerados satisfatórios. Neste trabalho, para o primeiro ano de avaliação e para este parâmetro todos os valores ou se aproximaram ou foram superiores a este valor, demonstrando assim que as respostas obtidas pelo método não destrutivo são confiáveis e podem ser utilizados para predizer os valores de sólidos solúveis em pêssegos.

Tabela 3. Validação das equações de calibração para sólidos solúveis e firmeza de polpa $\left({ }^{\circ} \mathrm{Brix}\right.$ e $\left.\mathrm{kgf} / \mathrm{cm}^{2}\right)$, através dos parâmetros estatísticos R²(Coeficiente de Determinação), SEP (Erro Padrão dos Valores Preditos, em ${ }^{\circ}$ Brix e kgf/ $\left.\mathrm{cm}^{2}\right)$ e as equações de reta para o modelo de regressão linear $(\mathrm{y}=\mathrm{ax}+\mathrm{b})$ relativos as validações das calibrações para a determinação de sólidos solúveis e firmeza de polpa em pêssegos Chimarrita, Maciel, Eldorado e Jubileu (n: 50 frutas) para o ano de 2009. UFPel, Pelotas,RS, 2013.

\begin{tabular}{llcc}
\hline \multicolumn{1}{c}{ Cultivar } & \multicolumn{2}{c}{ Validação/ Sólidos Solúveis } & \\
\hline Chimarrita & $0,82^{* *}$ & SEP & Equação \\
Maciel & 0,84 & 0,85 & $\mathrm{y}=0,75 \mathrm{x}+2,42$ \\
Eldorado & 0,78 & 2,36 & $\mathrm{y}=1,16 \mathrm{x}-3,66$ \\
Jubileu & 0,69 & 0,92 & $\mathrm{y}=1,07 \mathrm{x}-1,16$ \\
\hline \multicolumn{4}{c}{ Validação/ Firmeza de polpa } \\
\hline Chimarrita & $0,40^{* *}$ & 1,61 & $\mathrm{y}=0,78 \mathrm{x}+2,85$ \\
Maciel & 0,16 & 1,23 & $\mathrm{y}=0,59 \mathrm{x}+2,92$ \\
Eldorado & 0,63 & 3,34 & $\mathrm{y}=0,40 \mathrm{x}+1,51$ \\
Jubileu & 0,63 & 2,18 & $\mathrm{y}=0,79 \mathrm{x}+3,21$ \\
\hline
\end{tabular}

$* * \mathrm{p}=0,001$.

Fonte: Elaboração dos autores. 
Para sólidos solúveis, observou-se os menores valores de SEP para as amostras referentes a cultivar Jubileu, sendo de $0,82^{\circ}$ Brix, e os maiores valores para a cultivar Maciel com 2,36 ${ }^{\circ}$ Brix (Tabela 3). Este parâmetro permite a comparação com outras equações de calibração para a estimativa de um mesmo componente e a adequabilidade de um modelo de predição pode ser avaliada quando se obtém baixos valores de SEP e altos valores de $\mathrm{R}^{2}$ (ROGGO et al., 2007)

Para a firmeza de polpa, não foram observados valores adequados de coeficientes de determinação para as cultivares analisadas no primeiro ano (Tabela 3), sendo os coeficientes de determinação de 0,40 para a cultivar Chimarrita, 0,16 para a cultivar Maciel e 0,63 para as cultivares Eldorado e Jubileu. Embora estas duas últimas cultivares apresente um maior valor para este coeficiente, os altos valores de SEP determinados, indicam que o modelo pode não ser adequado para análises futuras.

Os dados obtidos para o parâmetro sólidos solúveis, no primeiro ano, estão de acordo com os autores Shao, Bao e He (2011) no qual determinaram o conteúdo de açúcares em duas variedades de pêssegos "Milu e Hongxianjiu" utilizando um equipamento Vis/Nir (400-1000nm) e processamento dos dados através de mínimos quadrados parciais, obtiveram $\mathrm{R}^{2}$ de 0,94 , ao passo que Golic e Walsh (2006) obtiveram calibração para sólidos solúveis, através de PSL, em frutas de caroços (pêssegos, nectarinas e ameixas), com valores de coeficiente de determinação de 0,80 , para as três variedades juntas utilizando espectros entre 735 e $932 \mathrm{~nm}$.

A previsão de um parâmetro de natureza física, como a firmeza de polpa, está relacionada à perda das estruturas da parede celular, como pectinas, celulose e hemicelulose e estes compostos possuem diferentes picos de absorção influenciando os espectros gerados (PAZ et al., 2008). Associado a isto, um dos grandes pontos críticos existentes nos modelos de calibrações para este parâmetro é a obtenção dos métodos de referência pouco precisos e, neste caso, Nicolaï et al. (2006) sugerem o uso de métodos de impacto ou de vibração para a determinação desta característica em processos de calibração.

Para o segundo ano de avaliação, os resultados das calibrações realizadas nas diferentes datas de colheita, apresentaram valores de coeficientes de regressão múltipla inferiores ao reportados no primeiro ano de avaliação (Tabela 4). Observa-se que para as amostras que compuseram as equações de calibração, os valores médios foram maiores para sólidos solúveis e firmeza de polpa, do que os reportados no ano anterior (Tabela 2), este fato pode ser atribuído à menor ocorrência de precipitação na época da colheita da safra de 2010 em relação a 2009. Entretanto, como foi incluída a calibração do ano anterior para a geração dos novos espectros, esta não possuía um intervalo que abrangesse os novos valores que originaram as amostras do ano de 2010 podendo ser esta uma das causas dos baixos valores comparados entre as safras.

Os melhores valores foram identificados na cultivar Jubileu na qual foram determinados coeficientes de regressão múltipla $\left(\mathrm{R}^{2}\right)$ satisfatórios entre 0,83 a 0,95 para sólidos solúveis e 0,65 a 0,93 para a firmeza de polpa, em todas as datas de colheita. A influência do fator ano em calibrações já foi relatada em trabalho de Peirs et al. (1998), onde os autores estudaram o efeito do ano e da cultivar sobre os modelos de calibração usando a espectroscopia em comprimentos de onda compreendidos entre 800-1500nm para a análise de sólidos solúveis em pêssegos, os autores descrevem que a combinação de diferentes anos e diferentes cultivares na mesma calibração apresenta valores de coeficientes que variam de 0,92 a 0,97 dependendo da combinação utilizada e SEC de 0,4 a 1,63 ${ }^{\circ}$ Brix concordando com os valores encontrados neste trabalho. 
Tabela 4. Datas de colheita, valores médios (expressos ${ }^{\circ}$ Brix para solidos soluveis e $\mathrm{kgf} / \mathrm{cm}^{2}$ para firmeza de polpa), coeficiente de regressão múltipla $\left(\mathrm{R}^{2}\right)$ e erro padrão de calibração (SEC) para as curvas de calibração geradas pelo software NCS para as amostras de pêssegos das cultivares Chimarrita, Maciel, Eldorado e Jubileu ( n: 350 frutas) para o ano de 2010. UFPel, Pelotas,RS, 2013.

\begin{tabular}{|c|c|c|c|c|c|c|c|}
\hline \multicolumn{8}{|c|}{ Parâmetros estatísticos da Calibração } \\
\hline \multicolumn{5}{|c|}{ Sólidos solúveis } & \multicolumn{3}{|c|}{ Firmeza de polpa } \\
\hline Cultivar & Datas & Médias & $\mathbf{R}^{2}$ & SEC & Médias & $\mathbf{R}^{2}$ & SEC \\
\hline \multirow{3}{*}{ Chimarrita } & $07 / 12 / 10$ & 12,5 & $0,63 * *$ & 1,0 & 3,5 & $0,41 * *$ & 1,6 \\
\hline & $10 / 12 / 10$ & 12,9 & 0,60 & 0,9 & 4,6 & 0,73 & 0,8 \\
\hline & $14 / 12 / 10$ & 13,1 & 0,65 & 1,0 & 3,2 & 0,84 & 1,0 \\
\hline \multirow{3}{*}{ Maciel } & $20 / 12 / 10$ & 13,1 & 0,70 & 0,8 & 2,7 & 0,63 & 0,8 \\
\hline & $23 / 12 / 10$ & 13,5 & 0,91 & 0,6 & 2,7 & 0,40 & 0,8 \\
\hline & $27 / 12 / 10$ & 13,2 & 0,82 & 0,6 & 2,6 & 0,21 & 0,6 \\
\hline \multirow{4}{*}{ Eldorado } & $19 / 12 / 10$ & 11,5 & 0,62 & 0,8 & 5,2 & 0,33 & 1,2 \\
\hline & $24 / 12 / 10$ & 12,3 & 0,50 & 1,0 & 4,6 & 0,40 & 1,2 \\
\hline & $28 / 12 / 10$ & 12,6 & 0,64 & 0,7 & 3,7 & 0,63 & 1,0 \\
\hline & $05 / 01 / 11$ & 13,6 & 0,65 & 0,9 & 1,3 & 0,59 & 0,9 \\
\hline \multirow{4}{*}{ Jubileu } & $19 / 12 / 10$ & 11,7 & 0,92 & 0,4 & 3,1 & 0,93 & 0,5 \\
\hline & $24 / 12 / 10$ & 12,0 & 0,95 & 0,3 & 1,8 & 0,71 & 0,4 \\
\hline & $28 / 12 / 10$ & 12,0 & 0,83 & 0,6 & 1,3 & 0,65 & 0,3 \\
\hline & $05 / 01 / 11$ & 12,8 & 0,95 & 0,5 & 1,6 & 0,92 & 0,2 \\
\hline
\end{tabular}

$* * \mathrm{p}=0,001$

Fonte: Elaboração dos autores.

Para a validação das calibrações para o ano de 2010 e os resultados obtidos estão apresentados na tabela 5. Na cultivar Chimarrita os valores dos coeficientes de determinação $\mathrm{R}^{2}$ variaram ao longo da maturação de 0,64 a 0,74 e SEP de 1,01 e 0,46 ${ }^{\circ}$ Brix para sólidos solúveis e 0,56 a 0,62 para a firmeza da polpa com SEP de 1,98 a $0,97 \mathrm{kgf} / \mathrm{cm}^{2}$, em comparação ao primeiro ano as equações de calibração para sólidos solúveis diminuíram a sua capacidade de prever resultados, entretanto para o parâmetro firmeza de polpa os resultados obtidos foram mais satisfatórios em comparação ao ano anterior, este resultado pode ter sido influenciado pelo aumento no número de amostras para compor o conjunto de calibração.

Para a cultivar Maciel os valores das calibrações para sólidos solúveis se mantiveram ajustados nas três datas de colheita utilizadas com valores de $\mathrm{R}^{2}$ variando de 0,83 a 0,86 e SEP 0,93 a $1,18^{\circ}$ Brix, para o parâmetro firmeza de polpa os valores dos coeficientes de determinação variam de 0,17 a 0,51 com SEP de 0,98 a $0,62 \mathrm{kgf} / \mathrm{cm}^{2}$, no qual são considerados baixos e não satisfatórios para a análise de amostras futuras. 
Tabela 5. Parâmetros estatísticos $\mathrm{R}^{2}$ (Coeficiente de Determinação), SEP (erro padrão dos valores preditos, em ${ }^{\circ}$ Brix para solidos soluveis e $\mathrm{kgf} / \mathrm{cm}^{2}$ para firmeza de polpa) e a equação da reta para o modelo de regressão linear $(\mathrm{y}=\mathrm{ax}+\mathrm{b})$ relativos as validações das calibrações, realizadas em diferentes datas de colheita para a determinação de sólidos solúveis e firmeza de polpa em pêssegos Chimarrita, Maciel, Eldorado e Jubileu (n: 100 frutas), para o ano de 2010. UFPel, Pelotas,RS, 2013.

\begin{tabular}{|c|c|c|c|c|c|c|c|}
\hline \multirow{3}{*}{ Cultivar } & \multicolumn{7}{|c|}{ Validação } \\
\hline & \multirow[b]{2}{*}{ Datas } & \multicolumn{3}{|c|}{ Sólidos solúveis ( ${ }^{\circ}$ Brix) } & \multicolumn{3}{|c|}{ Firmeza de polpa $\left(\mathrm{kgf} / \mathrm{cm}^{2}\right)$} \\
\hline & & $\mathbf{R}^{2}$ & SEP & Equação & $\mathbf{R}^{2}$ & SEP & Equação \\
\hline & $07 / 12 / 10$ & $0,66 * *$ & 0,74 & $y=0,67 x+3,54$ & $0,56 * *$ & 1,98 & $y=0,61 x+0,07$ \\
\hline \multirow[t]{2}{*}{ Chimarrita } & $10 / 12 / 10$ & 0,64 & 0,67 & $y=0,74 x+2,75$ & 0,54 & 1,47 & $y=0,48 x+1,21$ \\
\hline & $14 / 12 / 10$ & 0,74 & 0,46 & $y=0,76 x+2,77$ & 0,62 & 0,97 & $y=0,59 x+1,90$ \\
\hline \multirow{4}{*}{ Maciel } & Datas & $\mathbf{R}^{2}$ & SEP & Equação & $\mathbf{R}^{2}$ & SEP & Equação \\
\hline & $20 / 12 / 10$ & 0,86 & 1,18 & $y=0,88 x+2,45$ & 0,17 & 0,98 & $y=0,24 x+1,64$ \\
\hline & $23 / 12 / 10$ & 0,83 & 1,32 & $y=0,81 x+3,48$ & 0,34 & 0,69 & $y=0,38 x+1,62$ \\
\hline & $27 / 12 / 10$ & 0,85 & 0,92 & $y=0,71 x+4,15$ & 0,51 & 0,62 & $y=0,53 x+1,04$ \\
\hline \multirow{5}{*}{ Eldorado } & Datas & $\mathbf{R}^{2}$ & SEP & Equação & $\mathbf{R}^{2}$ & SEP & Equação \\
\hline & $19 / 12 / 10$ & 0,72 & 0,82 & $y=0,78 x+2,44$ & 0,54 & 0,92 & $y=0,64 x+1,31$ \\
\hline & $24 / 12 / 10$ & 0,72 & 0,94 & $y=0,74 x+4,07$ & 0,57 & 0,99 & $y=0,57 x+0,55$ \\
\hline & $28 / 12 / 10$ & 0,76 & 0,86 & $y=0,76 x+2,61$ & 0,56 & 0,93 & $y=0,94 x+0,35$ \\
\hline & $05 / 01 / 11$ & 0,85 & 0,57 & $y=0,72 x+3,79$ & 0,68 & 1,03 & $y=0,84 x-0,34$ \\
\hline \multirow{5}{*}{ Jubileu } & Datas & $\mathbf{R}^{2}$ & SEP & Equação & $\mathbf{R}^{2}$ & SEP & Equação \\
\hline & $19 / 12 / 10$ & 0,65 & 0,57 & $y=0,68 x+4,48$ & 0,34 & 1,23 & $y=0,49 x+2,16$ \\
\hline & $24 / 12 / 10$ & 0,82 & 0,48 & $y=0,68 x+4,20$ & 0,41 & 0,79 & $y=0,29 x+2,03$ \\
\hline & $28 / 12 / 10$ & 0,70 & 0,58 & $y=0,72 x+3,50$ & 0,19 & 0,83 & $y=0,20 x+1,20$ \\
\hline & 05/01/11 & 0,77 & 0,70 & $y=0,76 x+3,53$ & 0,72 & 0,63 & $y=0,94 x-0,26$ \\
\hline
\end{tabular}

$* * \mathrm{p}=0,001$

Fonte: Elaboração dos autores.

Os valores obtidos para as validações das calibrações com as cultivares Eldorado e Jubileu apresentaram resultados que variaram de $R^{2} 0,72$ a 0,85 e 0,65 a 0,82 respectivamente para o parâmetro sólidos solúveis e para o parâmetro firmeza de polpa foram observados bons coeficientes de determinação sendo os melhores resultados de 0,68 para a cultivar Eldorado e 0,72 para a cultivar Jubileu, nestas duas cultivares os valores de SEP para os dois parâmetros são considerados aceitáveis se comparados com demais autores já citados anteriormente (Tabela 5).

Embora os coeficientes de determinação não sejam elevados para a firmeza da polpa, há uma tendência de melhores capacidades de previsão nas últimas datas de colheita, fato este observado em todas as cultivares em estudo (Tabela 5), isto pode ser explicado pela maior heterogeneidade das frutas no final da colheita, apresentando frutas extremamente maduras como frutas ainda verdes contemplando todas as classes de maturação, um dos requisitos principais para o sucesso no desenvolvimento de calibrações.

Outro ponto a discutir em relação aos melhores resultados da firmeza de polpa no segundo ano diz respeito ao maior número de amostras utilizadas nas calibrações em comparação ao primeiro ano e desta forma a ampliação do banco de dados que compõe os modelos. Segundo Wiedemann et al. (1998) o número de amostras, o intervalo de concentrações coberto pelas amostras e a distribuição dentro desse intervalo é de extrema importância para a aquisição de boas calibrações. O número de amostras utilizadas neste trabalho é considerado satisfatório quando comparados a outras bibliografias onde se pode encontrar amostras compostas por 340 (VENTURA et al., 1998), 200 (COSTA; NOFERINI; 
ANDREOTTI, 2002), 420 frutas (NICOLAÏ et al., 2008), 40 frutas (GABIOUD et al., 2008) e 80 (LIU; CHEN; OUYANG, 2008). Alamar et al., 2007 cita que as principais causas que devem ser consideradas na calibração dos equipamentos são: mudanças nos comportamentos físicos das amostras (composição química de acordo com as variedades), mudança na resposta instrumental (referentes aos equipamentos), assim como a precisão das análises de referência.

\section{Conclusões}

A época de colheita das frutas influencia nos modelos de calibração para sólidos solúveis e firmeza de polpa, obtendo-se melhores ajustes ao final da safra.

De acordo com a validação das calibrações para sólidos solúveis em pêssegos das cultivares Chimarrita, Maciel, Eldorado e Jubileu, foi possível estabelecer modelos de calibração confiáveis para a estimativa deste parâmetro em amostras futuras com o uso da espectroscopia Vis/NIR.

Para a firmeza de polpa, não foram obtidos modelos confiáveis para estimar este parâmetro no primeiro ano de avaliação. Para o segundo ano, apenas os modelos desenvolvidos para as cultivares Jubileu e Eldorado foram considerados satisfatórios.

\section{Referências}

ALAMAR, M. C.; BOBELYN, E.; LAMMERTYN, J.; NICOLAÏ, B. M.; MOLTÓ, E. Calibration transfer between NIR diode array and FT-NIR spectrophotometers for measuring the soluble solids contents of apple. Postharvest Biology and Technology, Amsterdam, v. 45, n. 1, p. 38-45, 2007.

BEEBE, K. R.; PELL, R. J.; SEASHOLTZ, M. B. Chemometrics: a practical guide. New York: J. Wiley, 1998. p. 46-47.

BOBELYN, E.; SERBAN, A. S.; NICU, M.; LAMMERTYN, J.; NICOLAI, M. B.; SAEYS, W. Postharvest quality of apple predicted by NIRspectroscopy: study of the effect of biological variability on spectra and model performance. Postharvest Biology and Technology, Amsterdam, v. 55, n. 1, p. 133-143, 2010.

COSTA, G.; ANDREOTTI, C.; MISEROCCHI, O.; NOFERINI, M.; SMITH, G. S. Near-Infrared (NIR) methods to determine kiwifruit field harvest date and maturity parameters in cool-store. IVth Int. Symposium on kiwifruit, Santiago (Chile), January 11-14. Acta Horticulturae, Leuven, v. 498, n. 1, p. 231-37, 1999.

COSTA G.; NOFERINI, M.; ANDREOTTI, C. Non-destructive determination of internal quality in intact pears by near infrared spectroscopy. In: INTERNATIONAL SYMPOSIUM ON PEAR, 8., 2002, Bologna. Proceedings... Bologna: Acta Horticulture, v. 596, 2002. p. 821-825.

GABIOUD, S.; BAUMGARTNER, D.; GASSER, F.; KNEUBÜHLER, Y.; LATTMANN, S.; HOHN, E. Non destructive quality measurements on apples. Acta Horticulture, Leuven, v. 1, n. 796, p. 217-224, 2008.

GOLIC, M.; WALSH, K. B. Robustness of calibration models based on near infrared spectroscopy for the inline grading of stonefruit for total soluble solids content. Analytica Chimica Acta, Oxford, v. 555, n. 1, p. 286-291, 2006.

GUTHRIE, J. A.; WEDDING, B.; WALSH, K. B. Robustness of NIR calibrations for soluble solids in intact melon and pineapple. Journal Near Infrared Spectroscopy, Austrália, v. 6, n. 1, p. 259-265, 1998.

HUANG, H.; YU, H.; XU, H.; YING, Y. Near infrared spectroscopy for on/in-line monitoring of quality in foods and beverages: a review. Journal of Food Engineering, Essex, v. 87, n. 1, p. 303-313, 2008.

LIN, Y.; YING, Y. Theory and application of near infrared spectroscopy in assessment of fruit quality: a review. Sensor \& Instrument Food Quality Safety, Nova York, v. 3, n. 1, p. 130-141, 2009.

LIU, Y.; CHEN, W.; OUYANG, A. Nondestructive determination of pear internal quality indices by visible and near-infrared spectrometry. Food Science and Technology, Espanha, v. 1, n. 48, p. 1720-1725, 2008.

MCGLONE, V. A.; KAWANO, S. Firmness, dry-matter and soluble-solids assessment of postharvest kiwifruit by NIR-spectroscopy. Postharvest Biology Technology, Amesterdam, v. 13, n. 1, p. 131-141, 1998.

NICOLAÏ, B. M.; BEULLENS, K.; BOBELYN, E.; HERTOG, M.; SCHENK, A.; VERMEIR, S.; LAMMERTYN, J. Systems to characterize internal quality of fruit and vegetables. In: INTERNATIONAL CONFERENCE ON MANAGING QUALITY IN 
CHAINS - THE INTEGRATED VIEW ON FRUITS AND VEGETABLES QUALITY, 4., 2006, Bangkok. Proceedings... Bangkok: Acta Horticulture, 2006. p. 5965.

NICOLAÏ, B. M.; VERLINDEN, B. E.; DESMET, M.; SAEVELS, S.; SAEYS, W.; THERON, K.; CUBEDDU, R.; PIFFERI, A.; TORRICELLI, A. Time-resolved and continuous wave NIR reflectance spectroscopy to predict soluble solids content and firmness of pear. Postharvest Biology Tecnology, Amsterdam, v. 47, n. 1, p. 68-74, 2008.

NOFERINI, M.; FIORI, G.; ZIOSI, V.; COSTA, G. L'indice DA, un nuovo parametro non distruttivo per stabilire l'epoca di raccolta e la qualità del frutto di pesco. Rivista di Frutticoltura e di Ortofloricoltura, Bologna, v. 1, n. 7-8, p. 26-29, 2008.

PAZ, P.; SÁNCHEZ, M. T.; PÉREZ-MARÍN, D.; GUERRERO, J. E.; GARRIDO-VARO, A. Nondestructive determination of total soluble solids content and firmness in plums using near-infrared reflectance spectroscopy. Journal Agriculture Food Chemistry, Davis, v. 56, n. 1, p. 2565-2570, 2008.

PEIRS, K. H. S.; DELL, G. G.; LEFFLER, R. G.; KAY, S. J. Near infrared spectrometric method for nondestructive determination of soluble solids content of peaches. Journal American Society Horticulture Science, Virginia, v. 123, n. 5, p. 808-905, 1998.

PEIRS, A.; SCHEERLINCK, N.; TOUCHANT, K.; NICOLAÏ, B. M. Comparison of fourier transform and dispersive near-infrared reflectance spectroscopy for apple quality measurements. Biosystens Engineering, Amesterdam, v. 81, n. 3, p. 305-311. 2002.
ROGGO, Y.; CHALUS, P.; MAURER, L.; LEMAMARTINEZ, C.; EDMOND, A.; JENT, N. A review of near infrared spectroscopy and chemometrics in pharmaceutical technologies - review. Journal of Pharmaceutical and Biomedical Analysis, Amesterdam, v. 44, n. 1, p. 683-700, 2007.

SCARITPORN, V.; ZHAO, J.; CHEN, Q.; RACHATA, C. Application of FT-NIR spectroscopy to the measurement of fruit firmness of "Fuji" apples. International Journal of Science and Technology, Reino Unido, v. 2, n. 1, p. 13-23, 2008.

SHAO, Y.; BAO, Y.; HE, Y. Visible/near-infrared spectra for linear and nonlinear calibrations: a case to predict soluble solids contents and $\mathrm{pH}$ value in peach. Food Bioprocess Technology, Nova York, v. 4, n. 8, p. 13761383, 2011.

VENTURA, M.; JAGER, A.; PUTTER, H.; ROELOFS, F. P. M. M. Non-destructive determination of soluble solids in apple fruit by near infrared spectroscopy (NIRS). Postharvest Biology Technology, Amsterdam, v. 14, n. 1, p. 21-27, 1998.

WIEDEMANN, S. C. C. C.; HANSEN, W. G.; SNIEDER, M.; WORTEL, V. A. L. NIR calibration in practice. Analusis Magazine, v. 26, n. 4, p. M38-M43, 1998.

ZERBINI, P. E. Emerging technologies for nondestructive quality evaluation of fruit. Journal of Fruit and Ornamental Plant Research, Filadélfia, v. 14, n. 2, p. 13-23, 2006. 